Cahiers de géographie du Québec

\title{
Le redéploiement spatial de la production : une voie de relance de l'artisanat traditionnel au Maroc?
}

Le cas de la dinanderie à Fès

\section{Relocation of production as a Way to Revitalize Traditional}

Crafts in Morocco

\section{A Study of Brassware Crafts in Fez}

El redespliegue espacial de la producción: ¿Es una vía de reactivación del artesanado tradicional en Marruecos?

\section{El caso de la latonería en Fes}

\section{Naoual Ouazzani Touhami, Améziane Ferguene et Larbi Jaidi}

Volume 59, numéro 167, septembre 2015

Version originale soumise en janvier 2015. Version révisée reçue en octobre 2015

URI : https://id.erudit.org/iderudit/1036353ar

DOI : https://doi.org/10.7202/1036353ar

Aller au sommaire du numéro

Éditeur(s)

Département de géographie de l’Université Laval

ISSN

0007-9766 (imprimé)

1708-8968 (numérique)

Découvrir la revue

Citer cet article

Ouazzani Touhami, N., Ferguene, A. \& Jaidi, L. (2015). Le redéploiement spatial de la production : une voie de relance de l'artisanat traditionnel au Maroc ? Le cas de la dinanderie à Fès. Cahiers de géographie du Québec, 59(167), 167-188. https://doi.org/10.7202/1036353ar

\section{Résumé de l'article}

Cet article traite de l'évolution de l'artisanat de Fès, des difficultés auxquelles cet artisanat est confronté depuis plusieurs décennies et des conditions requises pour sa redynamisation. À l'évidence, pour retrouver son rayonnement d'antan, l'artisanat traditionnel doit opter pour l'innovation comme moyen privilégié de création de valeur, ainsi que pour un nouveau mode d'organisation de sa production. La politique de transfert des ateliers de la vieille ville vers de nouvelles " zones d'activités artisanales ", créées hors médina, est-elle une voie appropriée pour une telle redynamisation ? En d'autres termes, ce redéploiement spatial des activités fournit-il aux artisans l'occasion d'évoluer vers des formes de travail et d'organisation plus efficaces et plus compétitives ? Telle est la problématique centrale qu'aborde ce texte. 


\title{
Le redéploiement spatial de la production: une voie de relance de l'artisanat traditionnel au Maroc?
}

\section{Le cas de la dinanderie à Fès}

Relocation of production as a Way to Revitalize Traditional Crafts in Morocco: A Study of Brassware Crafts in Fez

El redespliegue espacial de la producción: ¿Es una vía de reactivación del artesanado tradicional en Marruecos? El caso de la latonería en Fes

\author{
Naoual OUAZZANI TOUHAMI \\ Université Mohammed V de Rabat \\ nawalot@gmail.com \\ Améziane FERGUENE \\ Université Grenoble Alpes, PACTE-CNRS, F-38100 \\ ameziane.ferguene@upmf-grenoble.fr \\ Larbi JAIDI \\ Université Mohammed V Rabat-Agadal \\ jaidi@mtds.com
}

\section{Résumé}

Cet article traite de l'évolution de l'artisanat de Fès, des difficultés auxquelles cet artisanat est confronté depuis plusieurs décennies et des conditions requises pour sa redynamisation. À l'évidence, pour retrouver son rayonnement d'antan, l'artisanat traditionnel doit opter pour l'innovation comme moyen privilégié de création de valeur, ainsi que pour un nouveau mode d'organisation de sa production. La politique de transfert des ateliers de la vieille ville vers de nouvelles "zones d'activités artisanales», créées hors médina, est-elle une voie appropriée pour une telle redynamisation? En d'autres termes, ce redéploiement spatial des activités fournit-il aux artisans l'occasion d'évoluer vers des formes de travail et d'organisation plus efficaces et plus compétitives? Telle est la problématique centrale qu’aborde ce texte.

\section{Mots-clés}

Artisanat traditionnel, médina, nouvelles zones d'activités artisanales, politique de délocalisation, dynamique entrepreneuriale, savoir-faire anciens.

\begin{abstract}
Our article analyzes the evolution of Fez's handicraft sector, the challenges it has faced for many decades and the conditions needed to revitalize it. Clearly, to recover its former strength and influence, the traditional crafts sector in Fez needs to embrace innovation as the most effective way to enhance value, while also finding new ways to organize craft production. Is the transfer policy that moves existing craftshops from the old city to newly created "areas of craft activities» outside the medina the most appropriate way to achieve this revitalization? In other words, does the craftshops relocation policy create conditions whereby craftspeople can adopt more efficient and more competitive work practices? This is the key issue addressed in this article.
\end{abstract}




\section{Keywords}

Traditional crafts, medina, new craft activity areas, relocation policy, entrepreneurial dynamics, traditional know-how.

\section{Resumen}

Este artículo trata de la evolución artesanal en Fes, de las dificultades confrontadas desde hace decenios y de las condiciones necesarias a su redinamización. Evidentemente, para recuperar su lustre de antaño, el artesanado tradicional debe elegir la innovación como medio privilegiado para crear valor y como un nuevo modo de organización de su producción. ¿Es la política del traslado de los talleres de la vieja ciudad hacia las nuevas "zonas de actividades artesanales" creadas en las afueras de la medina una vía apropiada para tal redinamización? En otros términos ¿Es ese redespliegue espacial de actividades la oportunidad de evolucionar hacia formas de trabajo y de organización más eficientes y competitivas para los artesanos? Tal es la problemática central de este artículo.

\section{Palabras claves}

Artesanado tradicional, medina, nuevas zonas de actividades artesanales, política de delocalización, dinámica empresarial, técnicas antiguas.

\section{Introduction}

$\mathrm{Au}$ Maroc, les agglomérations artisanales, espaces où se perpétuent des activités manuelles ancestrales, s'observent dans les vieux centres urbains. Fès, notre terrain empirique de recherche, est parmi les villes du royaume où se concentrent d'anciennes et riches traditions artisanales, ainsi que des savoir-faire ancestraux transmis de génération en génération. L'artisanat est, aujourd'hui comme hier, l'une des principales activités économiques de la ville. Le dynamisme (relatif) de cette activité repose sur un socle de valeurs culturelles qui, en incitant les acteurs locaux à la coopération, favorise l'installation de petits artisans indépendants qui s'engagent dans l'aventure entrepreneuriale.

Toutefois, l'artisanat traditionnel ${ }^{1}$ «fassi» (c'est-à-dire de Fès) traverse de nos jours une phase délicate qui s'explique en partie par les difficultés d'adaptation aux évolutions de la demande mais aussi, pour une autre partie, par la concurrence des produits industriels importés. En outre, il présente des caractéristiques qui en font un secteur fragile malgré la richesse de ses savoir-faire anciens, profondément ancrés dans l'histoire de la ville. L'activité artisanale de la dinanderie, ${ }^{2}$ filière combinant

1 «Un artisanat traditionnel désignerait un métier à l'ancienneté attestée qui, dans son état actuel, ne serait pas radicalement différent de ce qu'il était autrefois. Il impliquerait le maintien d'une organisation en réseau caractérisée par une entraide elle-même héritée d'un ancien système de valeurs transmises de génération en génération» (Buob, 2009: 27).

2 La dinanderie est la fabrication artisanale d'objets en cuivre et, plus généralement, la production d'objets artisanaux par martelage de feuilles de métal. C'est une filière artisanale où la division du travail est très poussée, fondée sur des stratégies de spécialisation et de complémentarité. En effet, le processus de fabrication d'un produit dinandier (la théière ou le plateau, par exemple) nécessite l'intervention de plusieurs artisans, environ 17 spécialités. Chaque processus de production est exercé par un groupe d'artisans et délimite un métier spécifique. 
tradition et modernité (on y reviendra plus loin: 1.2.4), en fait partie. Par ailleurs, cette activité exercée en médina s'est révélée très polluante, constituant ainsi une menace inquiétante, tant pour la santé des artisans et du reste de la population que pour l'environnement et le devenir du métier artisanal lui-même. Remédier à cette menace est l'un des enjeux de la politique de transfert de cette activité nocive, de la médina vers la nouvelle zone Aïn Nokbi (créée à cet effet). L’autre enjeu crucial est naturellement de permettre à l'artisanat de Fès de renouer avec son rayonnement historique, grâce à la création de nouvelles activités sur la base des anciennes.

Au cœur de la réflexion proposée dans cet article, la question est donc: dans quelle mesure la politique de délocalisation de la filière de dinanderie vers la nouvelle zone Aïn Nokbi peut-elle induire une réorganisation de la production et un renouvellement des savoir-faire anciens et, sur cette base, favoriser une relance de la dynamique artisanale à Fès? Pour traiter cette question, nous avons procédé à une enquête sur le terrain. Ainsi, le travail empirique développé résulte en grande partie d'une enquête au moyen d'un questionnaire administré en face à face auprès de 80 artisans dinandiers localisés aussi bien dans le nouveau site Aïn Nokbi que dans la médina, de juillet 2013 à février $2014 .^{3}$ La diversité des unités productives ayant fait l'objet de l'enquête et la variété de leurs spécialités ont constitué un premier critère de représentativité de l'échantillon. L'autre critère est le caractère varié de ces unités selon la taille. Par ce travail, nous avons tenté de faire ressortir les dynamiques économiques, sociales et territoriales des espaces analysés.

Nous structurerons l'analyse selon un plan en deux parties. Dans un premier temps, nous présenterons le processus de transfert des activités de la vieille ville vers Aïn Nokbi. Dans un deuxième temps, nous nous efforcerons d'analyser les retombées de ce processus en termes de revitalisation du tissu productif artisanal sur le territoire de Fès (grâce, notamment, à un nouveau mode d'organisation qui assume le passage de l'artisanat à la petite industrie).

\section{Fès: mille ans d'évolution aboutissant au processus actuel de transfert des unités de production artisanales de la vieille ville vers les nouvelles zones d'activités}

Fès est considérée, à juste titre, comme un centre par excellence d'un savoir-faire artisanal remarquable, dont la pérennité est attribuable autant à la transmission continue de compétences manuelles d'une génération à l'autre qu'à des valeurs socioculturelles favorables. Toutefois, en raison de la saturation de l'espace de la vieille ville et des problèmes croissants d'insalubrité qui en découlent, l'État a lancé, au début des années 2000, une politique de transfert des activités artisanales vers de nouveaux sites. Cette politique, censée aider les artisans à s’adapter à la concurrence des produits industriels importés, a pour ambition stratégique de susciter un renouveau des métiers ancestraux et, au-delà, une redynamisation de l'économie ou, plus exactement, de la socioéconomie artisanale locale.

3 Voir l’annexe 1, qui récapitule les principaux éléments de cette enquête. 


\section{L'économie de Fès: survol historique d'un essor fondé sur un riche patrimoine artisanal}

\section{La construction de la vieille ville de Fès autour des activités artisanales}

Historiquement, Fès s'est développée en trois périodes: la partie la plus ancienne, «Fès El-Bâli», sous Idriss I et Idriss II, au VIIIe siècle; "Fès El-Jdid», au XIIIe siècle; et la ville européenne (dite «ville moderne»), sous le protectorat français, à partir de 1912. Au cours de ces différentes périodes, l’artisanat fassi a bénéficié des apports de trois cultures: berbère, andalouse et moyen-orientale, les trois portant d'une façon ou d'une autre (et à différents degrés) l'empreinte de la religion musulmane.

Les Berbères sont détenteurs d'un art artisanal séculaire qui se repère à quelques techniques particulières de fabrication. À Fès, l'influence berbère se manifeste aussi (surtout?) au niveau des institutions sociales incarnées dans l'organisation communautaire en Jemâa, dans laquelle le collectif prime sur l'individu. Au plan artisanal, l'élection de l'Amin à la tête de chaque corporation traduit un souci de représentativité de ses membres. Toutefois, l'influence musulmane y est aussi présente. Dès les IXe $\mathrm{X}^{\mathrm{e}}$ et XI ${ }^{\mathrm{e}}$ siècles, les apports de l'art islamique sont manifestes. Ils sont l'effet de l'installation au Maroc des Idrissides ${ }^{4}$ et des Béni Ifren. ${ }^{5}$ Ils seront plus sensibles encore avec les dynasties Almoravide (fin XI et début XII ${ }^{\mathrm{e}}$ siècles) et Almohade (XII e et XIII ${ }^{e}$ siècles), qui régnèrent sur un territoire allant du nord de l'Espagne jusqu'aux portes de l'Égypte et édifièrent d'imposants monuments en faisant appel à de nombreux artisans du bâtiment: maçons, plâtriers, zelligeurs, etc. Roger Le Tourneau (2009) met l'accent sur le rayonnement de l'artisanat fassi qui, selon lui, dépassait les limites du Maroc, s'étendant bien au-delà, en Algérie, Tunisie et Égypte, vers l'ouest, et au Sénégal, vers le sud.

Jusqu'à la fin de la Première Guerre mondiale, Fès avait une réelle importance sur le plan international; elle a développé des liens commerciaux intenses avec plusieurs villes en Europe, en Afrique et au Moyen-Orient. L'activité de production y était essentiellement le fait de corporations d'artisans que Louis Massignon (1925:84) définit comme «l'ensemble des maîtres ouvriers et apprentis exerçant dans la ville un même métier industriel ou commercial». C'est en leur sein que se prenaient les décisions concernant les métiers, que ce soit en matière économique, technique, sociale ou spatiale. Chacune de ces corporations était placée sous l'autorité d'un Amin. ${ }^{6}$ L'Amin est élu par les Maâllmines (les maîtres artisans) et eux seuls; une fois élu, il doit être reconnu par le Mouhtassib (le chef à l'échelle de plusieurs corporations). Une des missions principales de ces deux personnages (le Mouhtassib et l'Amin) est de régler les conflits qui peuvent survenir entre artisans et entre ceux-ci et leurs clients, afin d'en minimiser les effets défavorables. La bonne tenue de cette mission, au cours du temps, a permis d'assurer à Fès un climat stable, favorable à l'essor de l'activité artisanale.

4 Les Idrissides sont une dynastie arabe arrivée au Maroc au VIII siècle; Idriss $1^{\text {er }}$ (le fondateur), ayant fui Baghdad sous la menace des Abbassides, s’est installé à Walili (actuel Volubilis).

5 Les Béni Ifren sont une puissante famille berbère qui, à la tête des Zénètes, a édifié au VIII ${ }^{\mathrm{e}}$ siècle, sur le territoire correspondant aujourd'hui à l'extrême ouest de l'Algérie, un royaume, avec pour capitale Tlemcen.

6 L'Amin est le chef d'un métier. La racine «amn» du mot se réfère à la confiance, la loyauté, l'honnêteté, la sûreté. Issu du métier, l’Amin est élu par une assemblée d'artisans notables. 


\section{La Dynamique technicoéconomique de production sur le territoire de Fès}

Fès a de tout temps tenu une place singulière dans l'histoire du Maroc et apporté une contribution significative à l'économie du pays, grâce à la diversité des activités artisanales déployées sur son territoire et à leur réputation bien au-delà des frontières. Ces activités sont le fait de toute une constellation de petites unités de production regroupées géographiquement (sur l'espace restreint de la médina) selon une logique de proximité productive permettant une mutualisation des moyens, des échanges de biens et de services et, partant, des économies d'agglomération au profit des unités de production: prêts d'équipement, échanges de commandes, d'information, rencontres pour discuter de la meilleure façon d'améliorer la production...

Cette proximité a joué un rôle important dans le fonctionnement efficace du système artisanal local. En effet, d'une part, grâce à la division du travail et à la spécialisation qui s'en est trouvée facilitée, elle a favorisé une élévation continue de la productivité des artisans ; d'autre part, en incitant les unités de production artisanale à nouer entre elles des relations durables de coopération (notamment dans le cadre de contrats de sous-traitance), elle leur a constamment permis de bénéficier d'économies de coûts de transaction non négligeables. Bref, en autorisant la mise en commun des ressources, cette proximité a, de façon continue, autorisé des synergies et des externalités profitables à tous.

Sans doute, la proximité n’abolit-elle pas la concurrence entre entreprises locales produisant les mêmes biens. Toutefois, comme le montrent Xavier Greffe et Véronique Simonnet (2008) dans le cas des activités culturelles, l'effet de synergie (du regroupement géographique) l'emporte souvent sur l'effet de concurrence, qui s'avère néfaste surtout pour la survie des entreprises des métiers d'art s'adressant principalement à une clientèle locale et entre lesquelles le risque de copiage est élevé.

\section{Les valeurs socioculturelles locales au cœur de la régulation du système artisanal de la médina}

Si l'artisanat s'est développé à Fès surtout sur la base d'un savoir-faire ancestral et de ressources patrimoniales endogènes, ce développement doit beaucoup aussi au système de valeurs culturelles propres à cette ville. Celles-ci, partagées non seulement par les artisans mais par l'ensemble des acteurs locaux, ont joué (et jouent encore) un rôle de régulation institutionnelle de l'économie locale. Cela est vrai en particulier au sein des différentes corporations artisanales, dans lesquelles le comportement des unités de production est régulé de façon à toujours maintenir la concurrence dans certaines limites, et cela, en vertu de normes coutumières issues en partie de la hisba (institution musulmane qui édicte un ensemble de règles en matière de production et de commerce et prévoit un contrôle de leur bonne application au quotidien). Le respect de la hisba, est de la responsabilité du Mouhtassib, dont les arbitrages sont d'autant plus suivis d'effets que ses prérogatives lui sont conférées par Dahir (décret royal). Dans ces conditions, on comprend que le Mouhtassib (tout comme, à son niveau, l'Amin) ait de tout temps œuvré avec efficacité en faveur du respect des réglementations et des normes en matière de qualité des produits, tout en veillant par ailleurs à l'observation des coutumes qui protègent les acteurs économiques des excès de la concurrence. 
Pour importants qu'ils soient, l'encadrement de la concurrence et la qualité des produits ne sont pas les seuls aspects concernés par la régulation institutionnelle assurée par les valeurs socioculturelles locales. Au sein de la médina de Fès, comme sans doute dans d'autres espaces urbains anciens comparables (Sfax en Tunisie, par exemple), les valeurs issues de la tradition favorisent également une certaine solidarité entre les membres d'un même corps de métier ou d'une même profession. Cette solidarité entre unités de production, qui restent concurrentes par ailleurs, est d'autant plus effective qu'elle est encouragée par la configuration spatiale particulière de la médina. Celle-ci se caractérise par l'exiguïté de son espace et, donc, par une forte proximité entre les acteurs économiques qui y évoluent. Cela rend nécessaires, pour ne pas dire indispensables, les relations de coopération. Or, cela mérite d'être précisé, qu'elles soient voulues ou subies par les individus concernés, ces relations de coopération enracinées dans la tradition ont de tout temps, à Fès comme ailleurs, contribué à la vitalité de la socioéconomie locale. Comme nous le constations dans un travail précédent (Ferguène, 2001:279), "la socialité traditionnelle, là où elle est préservée, a plusieurs traductions avantageuses à la fois socialement et économiquement: transmission d'une génération à l'autre des savoir-faire anciens, perpétuation des liens de solidarité ancestraux au sein de la communauté locale, etc.».

\section{Le déclin progressif du système artisanal local depuis le début du $\mathrm{XX}$ siècle}

S’appuyant sur un riche héritage de savoir-faire ancestraux, l'artisanat a été, pendant des siècles, le moteur principal de la prospérité de Fès. La domination française, dans le cadre du régime du Protectorat en vigueur de 1912 à 1956, a sinon mis un terme à cet état de choses, du moins fortement contribué à amoindrir la dynamique. De fait, après l'instauration de ce régime, on assiste à la marginalisation du Mouhtassib et de l'Amin, dont le rôle de régulation était si crucial : l'essentiel de leurs attributions s'est trouvé dispersé entre divers organes et divers personnages, notamment le pacha. Et, selon un enchaînement logique, s'est ensuivi un déclin assez rapide de l'institution de la hisba, provoquant à son tour une disparition progressive mais inexorable de l'ensemble des règles en matière, notamment, de localisation des activités, de recrutement de la main-d'œuvre et de contrôle de la qualité du travail réalisé qui faisaient la force de la régulation de la socioéconomie de la vieille ville de Fès.

Cette époque est marquée, par ailleurs, par la concurrence d’articles manufacturés importés d'Europe (c'est-à-dire des produits similaires fabriqués industriellement), accentuant l'évolution défavorable de pans entiers de l'artisanat fassi. Cette concurrence est d'autant plus durement ressentie par les artisans de Fès que ces produits industriels européens n'envahissent pas seulement le marché domestique marocain, mais également les marchés traditionnels d'exportation (Algérie, Égypte, Sénégal...). Faute de pouvoir résister à cette concurrence, l'artisanat est entré dans un processus de déclin qui, pour avoir été graduel, n’en a pas moins été irréversible. De fait, ce phénomène souvent résumé par l'expression «assaut commercial du capitalisme européen », marque le début d'une nouvelle phase de l'histoire de l'artisanat de Fès, au cours de laquelle la réduction sensible de leurs revenus a conduit les artisans à abandonner progressivement les règles anciennes de coopération, avec comme résultat (dans les métiers les plus touchés), une détérioration des conditions de travail et une perte de qualité des produits. 
Au cours de ces dernières décennies, l'artisanat traditionnel fassi est confronté à d'autres problèmes. D'abord, on note la rude concurrence des produits industriels de substitution, provenant notamment de Chine. Bien que de qualité médiocre, ces biens présentent un avantage en termes de prix qui compense dans une large mesure cet inconvénient (notamment aux yeux des ménages à revenus modestes), causant ainsi pour les artisans de Fès une chute de leurs ventes, aussi bien sur les marchés locaux qu'étrangers. À cette difficulté s'ajoute le renchérissement des intrants que les entreprises artisanales ne peuvent pas répercuter sur leurs prix, justement à cause de cette concurrence. Ensuite, l'artisan fassi est pénalisé par les circuits d'approvisionnement et de commercialisation des matières premières et des produits finis, qu'il ne maîtrise pas et qui sont contrôlés par des grossistes et des «bazaristes». Enfin, ces difficultés de l'artisanat sont allées de pair, paradoxalement, avec une augmentation de l'effectif des artisans, qui se fait au détriment, d'une part, de la qualité des produits (qui ne cesse de se dégrader) et, d'autre part, de la protection de l'environnement (confronté à des problèmes graves et non pris en charge) .

Dernière remarque, mais non la moindre, le territoire de la médina de Fès est, depuis plusieurs années, confronté au phénomène dit de " déséconomies d'agglomération » 7 à cause de blocages liés à la configuration de l'espace et à l'exiguïté des locaux, aussi bien professionnels que privés: encombrement des ruelles, pollution et nuisances diverses liées à l'activité artisanale, etc. La proximité spatiale, qui était un avantage de la médina, semble se retourner en inconvénient entravant le bon fonctionnement du système économique local. Par ailleurs, l'environnement professionnel dans la vieille ville est sérieusement dégradé: les lieux d'exercice du métier sont devenus inadéquats, les règles d'hygiène et de sécurité sont trop souvent négligées et la législation de travail n'est pas respectée. De plus, une partie des activités artisanales de la médina est devenue une source majeure de pollution à travers les multiples effluents véhiculant des déchets toxiques et les divers métaux lourds rejetés dans la nature. Cet engrenage est arrivé à un point tel que certaines activités artisanales, réputées les plus nocives (à savoir la dinanderie, la poterie et la tannerie), sont prioritairement visées par la politique de transfert hors de la médina, une stratégie essentielle dans l'optique de la sauvegarde de la ville antique.

\section{Transfert des activités artisanales vers de nouvelles zones et renouveau des anciens métiers par la réactivation/réactualisation des savoir-faire locaux: la dinanderie à Aïn Nokbi}

Plusieurs types de métiers artisanaux font actuellement l'objet d'un transfert de la vieille ville vers de nouvelles zones d'activités artisanales (créées à cette fin à Aïn Nokbi, à Beljelik, etc.). Dans l'analyse qui suit, nous avons fait le choix de nous concentrer sur la dinanderie, et cela, pour deux raisons. D'une part, cette activité est très importante à Fès. Elle y tient la deuxième place, dans l'ensemble du secteur

7 Contraire des économies externes d’agglomération, qui signifie «les bénéfices que tire chaque entreprise du district du fait de son insertion dans une agglomération industrielle suffisamment grande» (Courlet et Pecqueur, 1996). 
artisanal, à la fois en termes de production, d'exportation et de population employée. ${ }^{8}$ D'autre part, la dinanderie est l'activité artisanale dans laquelle les innovations, à la fois de produits et de procédés, sont les plus manifestes, et les interactions avec l'industrie, les plus fortes. De plus, outre son rôle socioéconomique, la dinanderie se caractérise par une forte dimension culturelle, liée à sa capacité de transmettre un savoir-faire ancien. La majorité des unités de production dinandière étant en cours de délocalisation, le choix de centrer l'analyse sur cette activité vise à montrer en quoi elle correspond à un secteur économique dynamique et complexe, mais qui fonctionne désormais de plus en plus de façon mimétique, au sens où, sans renoncer totalement à la logique artisanale qui est historiquement la sienne, il cherche simultanément à s'approprier la logique «moderne» du secteur industriel.

\section{Le projet d'une zone industrielle à Aîn Nokbi pour accueillir les activités polluantes: rappel historique}

L'importance stratégique du transfert des activités polluantes hors de la médina est apparue dès les années 1970. Dans le schéma directeur de l'urbanisme de 1978, on considérait déjà ce transfert comme une mesure urgente dont la mise en œuvre était une condition de réussite du projet de sauvegarde de la médina. Lancé en 1979 par l'Office de développement industriel, le Programme national d'aménagement des zones industrielles contenait le projet d'une zone aménagée sur le site appelé Aïn Nokbi, pour accueillir les activités devant faire l'objet de la délocalisation. En 1982, la mission de coordination du projet était confiée à plusieurs acteurs ; puis, en 1992, l'Agence nationale de lutte contre l'habitat insalubre (ANHI) était désignée "comme opérateur, dans le cadre d'une convention de maîtrise d'ouvrage de l'opération de la zone Aïn Nokbi, pour acquérir et équiper le terrain support du projet». Cette convention a fixé le prix du mètre carré cessible à 750 dh (68 euros environ, un euro valant approximativement 11 dirhams sur le marché), prix alors considéré exorbitant par les artisans, et qui les a dissuadés de souscrire au projet. Cet obstacle a paralysé l'opération pendant plusieurs années.

La relance de ce projet, en 2000, par le ministère du Commerce et de l'Industrie (qui le subventionne en puisant dans le fonds Hassan II pour le développement économique et social), s'inscrit cette fois non pas dans le cadre du projet de sauvegarde de la médina, mais dans celui d'une zone d'activités génératrices d'emplois. C'est du reste ainsi qu'il a pu être subventionné par le fonds Hassan II pour le développement économique et social. Cette relance a été le fruit d'une concertation avec les acteurs locaux, qui a débouché sur une convention où sont définies clairement les "conditions de réalisation et d'achèvement de l'opération Aïn Nokbi», ainsi que les contributions des différents acteurs institutionnels. Les négociations ouvertes en 2002 entre l'ANHI (aujourd'hui Holding d'aménagement Al Omrane), la Délégation régionale de l'artisanat, les autorités locales et les artisans ont abouti à une baisse du prix du terrain: de 750 à 100 dh le mètre carré.

8 L'activité des métaux-et singulièrement la dinanderie-occupe la deuxième place au sein de l'artisanat fassi (1859 unités de production représentant $23 \%$ de l'ensemble du secteur artisanal), après celle du cuir et tissage (4364 unités, correspondant à 55\% du même secteur). Cette place est importante en comparaison notamment avec les autres activités artisanales, comme le bois (10\%) et la poterie $(3,6 \%)$. Le travail des métaux est aussi un grand pourvoyeur d'emplois: pas moins de 3000 personnes y sont occupées en permanence, soit 29\% de l'emploi artisanal (source: Statistiques de la Direction régionale de l'artisanat de Fès-Boulemane, 2011). Il est important, par ailleurs, pour sa forte contribution aux exportations de produits artisanaux. 
À la fin de l'année 2005, la Délégation régionale de l'artisanat de Fès a réalisé un recensement des artisans qui exercent des activités polluantes dans la médina pour en déterminer le nombre et les caractéristiques. En 2007, le projet Aïn Nokbi a été intégré dans le Plan de développement régional de l'artisanat, dont une partie est financée par le programme maroco-américain MCC (Millenium Challenge Corporation). ${ }^{9} \mathrm{Ce}$ projet s'inscrit également dans la vision stratégique élaborée par le gouvernement marocain pour l'artisanat, dite Vision 2015.

\section{La délocalisation de la dinanderie de la médina vers le site d'Ain Nokbi: la mise en œuvre concrète}

Le site Ä̈n Nokbi est un lotissement d'une superficie de 6,07 ha, équipé conjointement par l'administration centrale en charge de l'artisanat et les autorités communales de Fès, pour accueillir les unités de production de dinanderie déplacées de la médina. Ce site a été retenu essentiellement pour les avantages que procure sa proximité de la médina (3,5 km approximativement) et pour les facilités que présentent sa topographie et sa traversée par l'Oued Boukhrareb (qui permet le drainage des rejets liquides en aval de la ville).

Quelque 253 lots de terrain ont ainsi été attribués aux dinandiers de la médina par tirage au sort, en 2005. Sur ce nombre, 235 ont été attribués à des unités de production et 10 à des fournisseurs de matières premières. Trois autres ont été réservés à la construction de fondouks devant accueillir des sous-traitants; sur les cinq derniers lots, trois ont été mis à la disposition de trois organisations (une association, une coopérative et un laboratoire) pour y établir leur siège, et les deux autres servent respectivement de mosquée et de centre d'alphabétisation fonctionnelle. Précisons que 23 unités de production et 78 sous-traitants, qui étaient à l'origine dans la place Lalla Yedouna de la médina, ont été pris en charge par le programme MCC. Les artisans concernés ont aussi bénéficié d'indemnisations pour compenser les pertes subies du fait de leur déplacement involontaire. Les autres unités productives, construites par les artisans eux-mêmes, ont bénéficié d'un prix très bas du terrain, à $100 \mathrm{dh} / \mathrm{m}^{2}$, ainsi que d'une exonération du paiement de la taxe de lotir. Les artisans bénéficiaires se sont engagés, pour leur part, à prendre en charge le coût de construction de leurs ateliers, à respecter le cahier des charges d'attribution des lots de terrain et à localiser totalement leurs activités de production dans la nouvelle zone.

Aujourd'hui, c'est-à-dire plusieurs années après l'attribution des lots, le projet Aïn Nokbi piétine encore: au total, comme on vient de le dire, 235 lots ont été cédés à des unités de production; mais les travaux de construction ne sont achevés ou en cours d'achèvement que sur 155 lots. Et, sur ces derniers, seulement 123 unités de production sont effectivement en activité. Les autres demeurent toujours en médina. Quant aux fondouks, seul celui financé par le programme américano-marocain MCC a été livré aux bénéficiaires et est désormais opérationnel. Les deux autres, pris en charge par le Conseil de la région Fès-Boulmane et par le Conseil communal de Fès,

9 «Les États-Unis d'Amérique, agissant par le MCC, ont offert en 2007 un don au gouvernement marocain pour l'aménagement de la place Lalla Yedouna et la reconstruction et restructuration de quatre fondouks (Staouniyine, Al Barka, Chemaïne et Sbitriyine), situés tous au sein du tissu historique de la médina de Fès.» 
restent fermés, la négociation avec les autorités en vue d'un accès gratuit pour les sous-traitants bénéficiaires n'étant toujours pas bouclée. Il faut cependant noter que, grâce à la qualité des travaux d'aménagement réalisés dans le cadre du programme MCC en vue du déménagement des dinandiers de la place Lalla Yedouna (dans la médina) vers Ä̈n Nokbi, un réel engouement pour le nouveau site s'est fait sentir.

Outre la réduction de la pollution à Fès, l'enjeu de cette politique de délocalisation est triple. Elle vise en premier lieu à lever les contraintes diverses et variées qui pèsent sur l'activité économique dans la vieille ville. En second lieu, elle tend à soutenir la pérennité des métiers artisanaux, en offrant aux entreprises (déjà en activité comme à celles en cours de création) une zone de production adaptée à leurs besoins. En troisième lieu, moyennant des travaux de restauration, elle entend réhabiliter les sites historiques de la médina pour, notamment, y installer des ateliers spécialisés dans la finition des produits et qui serviront simultanément de points de vente, ceci afin de favoriser une meilleure articulation entre artisanat et tourisme et, par là même, d'accroître les revenus des artisans et de dynamiser les créations d'emplois dans le secteur.

\section{Appuis de l'État aux artisans confrontés aux multiples contraintes d'installation à Aïn Nokbi}

Pour aider les entreprises artisanales à surmonter les nombreuses contraintes liées à leur installation dans les nouvelles zones d'activités et à accéder aux services nécessaires à leur bon fonctionnement, différentes mesures ont été mises en œuvre par l'État. Dans le cas de Aïn Nokbi, ces mesures concernent notamment:

- le coût d'installation, qui a été étudié de façon à ce qu'il soit le moins élevé possible (entre autres avec l'exonération du paiement de la «taxe de lotir») ;

- la mise en place d'une cellule d'accompagnement durant la mise à exécution du projet, pour soutenir les artisans dans les opérations de déménagement sur le nouveau site (par exemple, minibus mis à la disposition des dinandiers pour le transfert de leurs machines);

- l'installation d'une station d'épuration des eaux usées qui, par le traitement approprié des résidus de la production, doit protéger l'environnement;

- l'exigence du respect de la réglementation du travail pour protéger la main-d'œuvre des excès souvent constatés dans le passé;

- l'instauration de dispositifs de garantie et de relais de financement pour soutenir la relocalisation et favoriser la pérennité des entreprises artisanales établies dans la nouvelle zone;

- l'amélioration des services aux entreprises sur le nouveau site, notamment en matière de conseil, d'entretien et de contrôle de la qualité;

- l'incitation au regroupement des activités (pour favoriser la mutualisation) et à la modernisation des points de vente (afin d'accroître leur attrait) ;

- la promotion de la qualité des produits par l'institution des labels et de leur protection. 


\section{La dinanderie à Aïn Nokbi: vers un modèle d'entreprise combinant tradition et modernité}

En déclin considéré comme irréversible, mais sans cesse renaissant, l'artisanat fassi se trouve aujourd'hui partagé entre deux conceptions: la première, restrictive, liée à une représentation ancestrale, synonyme de métiers anciens préservés dans leur authenticité, mais relativement dépassés d'un point de vue économique; et la seconde, extensive, liée à une image plus moderne des activités concernées, où l'artisan est vu d'abord comme un acteur économique qui, grâce à ses compétences, est à même de relever le défi de la concurrence, son atelier traditionnel cédant la place à une petite entreprise industrielle. Cette deuxième conception est très largement celle des artisans dinandiers désormais installés à Aïn Nokbi. En effet, tout en continuant à s'appuyer sur la tradition socioculturelle locale, les entreprises établies dans la nouvelle zone sont engagées dans un processus de modernisation de leurs activités: introduction de nouvelles machines dans la plupart des ateliers de production (en vue d'une mécanisation élevée), approfondissement de la division du travail (pour une plus grande productivité) et importation des matières premières prêtes à être utilisées. Ces facteurs ont eu des effets notables en termes de modernisation des techniques de fabrication. Cependant, les savoir-faire anciens ne sont pas niés; ils sont au contraire mobilisés pleinement, mais moyennant leur réactualisation pour adaptation aux conditions actuelles de la concurrence.

De notre travail de terrain, il ressort que les maîtres artisans sont en majorité des entrepreneurs d'âge mûr (à 67\%, ils ont entre 35 et 50 ans), ambitieux et ouverts sur le monde moderne. Beaucoup ont pris la relève de leurs pères ou de parents proches. Fait notable: même s'ils restent solidement enracinés dans la tradition, ils ont fait le choix d'une évolution de leurs méthodes de travail et de leur métier, ce qui veut dire qu'ils adoptent des moyens de production mécaniques, les locaux dans lesquels ils exercent à Aïn Nokbi (construits sur trois niveaux) étant plus spacieux et respectant les normes de sécurité et de santé au travail. Pour faire face à la concurrence des produits industriels, leurs entreprises disposent d'un atout non négligeable: une main-d'œuvre disponible localement en quantité, et peu exigeante sur le plan de la rémunération. En outre, cette main-d'œuvre fait souvent preuve d'une grande ingéniosité, cette qualité se manifestant notamment par sa capacité à utiliser du matériel de récupération, à reproduire diverses pièces de rechange (très chères ou difficiles à trouver sur le marché local) et à prolonger la durée de vie des machines au-delà de toute imagination (Ferguène, 2007). Enfin, elle montre une forte motivation sur le nouveau site de production qui offre, on l'a dit, de meilleures conditions de travail, en termes de normes de sécurité et de santé.

En définitive, le transfert des activités artisanales de dinanderie de la médina vers Aïn Nokbi s'accompagne d'une mutation de ces activités vers un modèle industriel. On assiste ainsi, sur le nouveau site, à un processus d' 'industrialisation de l'artisanat» (selon l'expression d'Ali Fejjal et de Driss Guerraoui, 1988), qui recouvre donc le passage (graduel) des unités de production concernées, d'un statut d'ateliers artisanaux traditionnels à celui de petites entreprises industrielles. En d'autres termes, la politique de transfert des activités, adoptée par les pouvoirs publics, a (intentionnellement ou non) induit un processus de développement industriel à petite échelle dans les nouvelles zones d'activité artisanale. D’un certain point de vue, 
au-delà d'une redynamisation ou relance de l'artisanat traditionnel, ce phénomène peut être interprété comme une régénération de cet artisanat, prenant la forme d'un développement, sur le nouveau site, de petites entreprises industrielles à partir d'ateliers artisanaux traditionnels issus de l'ancien site.

\section{Les effets dutransfert des activités de dinanderie en termes de développement de petites entreprises industrielles à partir des ateliers artisanaux traditionnels}

Grâce à la délocalisation d'une partie de ses entreprises de la médina vers le nouveau site, la filière de la dinanderie semble trouver un nouveau souffle. On observe, en effet, à Aïn Nokbi un regain de vitalité des unités de production concernées, sur la base d'une réactivation des anciens métiers et savoir-faire, ceux-ci étant désormais valorisés dans le cadre d'activités nouvelles, créées à travers un processus d'industrialisation des anciennes. En d'autres termes, le changement de site de production, parce qu'il va de pair avec la mutation d'une partie de l'activité dinandière de Fès (qui passe d'un statut artisanal à un statut industriel), s'accompagne d'une élévation globale de l'échelle de production du secteur et d'une amélioration des performances des entreprises installées sur le nouveau site. Comme tel, ce changement apparaît porteur d'un renouveau ou, tout au moins, d'une redynamisation de l'activité de dinanderie de Fès: redynamisation fondée sur la création-extension de petites entreprises industrielles à partir du socle artisanal préexistant. C'est cette idée que nous allons étayer en analysant les effets du transfert des activités à différents niveaux du vécu de la communauté locale.

\section{Les retombées sur le plan économique}

Sur le plan économique, la délocalisation des activités artisanales a plusieurs effets favorables sur le territoire de Fès. Le premier de ces effets apparaît, on l'a vu, sous la forme d'une bifurcation de l'artisanat traditionnel vers une logique industrielle entrepreneuriale, synonyme de multiplication des projets et de croissance de l'activité. Le second effet tient au redéploiement spatial de la production, qui se traduit par une extension de l'aire de diffusion des savoir-faire et une modernisation des méthodes de leur transmission dans le temps. Enfin, une troisième retombée se manifeste dans un souci croissant d'amélioration de la qualité, grâce notamment à l'innovation de produits et de procédés. Le résultat de l'action combinée de ces trois effets est sans équivoque: comme notre enquête de terrain l'a confirmé, l'activité de dinanderie connaît une reprise prometteuse dans la zone d'Aïn Nokbi alors que, dans le même temps, le processus de sa régression se poursuit dans la vieille ville.

\section{Une évolution défavorable en termes de nombre d'artisans et de production au sein de la médina}

Depuis que le projet de transfert a pris forme, le nombre d'artisans dinandiers exerçant dans la médina a diminué de plus de $50 \%$, ce qui met en évidence une perte au niveau d'une des principales «ressources spécifiques» de la socioéconomie de la vieille ville. 
Toutefois, ces acteurs n'ont pas disparu dans la nature, on les retrouve sur le nouveau site en tant qu'artisans ou petits industriels, toujours enracinés dans le territoire et ses traditions, mais plus ouverts désormais aux techniques modernes de production.

Quant à ceux qui continuent d'exercer leur métier dans la médina, ils enregistrent une forte baisse de leur activité. En effet, de l'amont à l'aval, la filière de la dinanderie se compose de toute une série de métiers, si bien que la fabrication d'un article nécessite plusieurs spécialités distinctes, mais complémentaires (pour certains articles, pas moins de 17 spécialités sont sollicitées). Le corollaire en est la prépondérance de la sous-traitance dans cette activité. L'habileté requise pour chacun de ces métiers rend improbable leur maîtrise par une seule personne ou leur intégration en totalité dans la même unité de production. D’où la réduction sensible de l'activité des unités qui continuent d'exercer dans la médina, à cause de la délocalisation de certaines composantes de la filière.

La délocalisation partielle n'est toutefois pas le seul facteur de dégradation de la production dinandière dans la médina. D’autres facteurs ont joué. On peut citer notamment la perte progressive du marché domestique marocain (en raison de la concurrence des produits industriels), ainsi que la fermeture de la frontière terrestre entre le Maroc et l'Algérie depuis 1994, qui a durement affecté l'économie fassie. En effet, les Algériens, les Libyens et les Tunisiens constituaient une partie substantielle de la clientèle. La fermeture de la frontière avec l'Algérie a fait chuter la demande émanant non seulement de ce pays mais aussi de Tunisie et de Libye, car, dans leur majorité, les clients maghrébins privilégiaient la voie terrestre. Cette donnée politique a donc eu des effets très préjudiciables pour les dinandiers de Fès et a entraîné une forte baisse de leurs revenus. Beaucoup d'unités de production ont été contraintes de cesser purement et simplement leur activité.

Pour illustrer les pertes engendrées au sein de la médina par le transfert des activités vers l'extérieur, il n'est que de considérer l'exemple des dinandiers de la Place Lalla Yedouna, dont les ateliers ont été délocalisés vers Aïn Nokbi. Ce déménagement a eu un impact négatif sur toute la vie du quartier entourant cette place: de nombreuses boutiques de produits de consommation courante et divers services (restaurants, cafés, etc.) ont dû fermer, et des petites activités familiales ont été mises en faillite, alors même que les populations concernées n’ont eu droit à aucune aide, ni accompagnement public.

\section{Mais une dynamique prometteuse de création-extension d'activités dans la nouvelle zone}

La dynamique à l'œuvre dans la nouvelle zone d'activités artisanales peut être analysée comme une bifurcation de l'artisanat traditionnel vers un artisanat (réactivé) entrepreneurial qui se fonde sur la création d'entreprises et leur extension progressive, à partir de la valorisation-revalorisation des savoir-faire ancestraux. À Aïn Nokbi, les dinandiers sont désormais des entrepreneurs évoluant dans un cadre formel alors qu'auparavant, dans la vieille ville, ils exerçaient en majorité dans des ateliers informels. En d'autres termes, dans la nouvelle zone, la légalité et la transparence sont de rigueur, ce qui se traduit par une concurrence de plus en plus forte entre unités de production fabriquant les mêmes biens. 
Par ailleurs, malgré la faiblesse de leurs moyens financiers, les perspectives de développement des entreprises sont bonnes, dans la mesure où les investissements nécessaires sont peu coûteux. À cet égard, il faut noter qu'à Ain Nokbi, grâce à un changement favorable du statut légal des locaux mis à leur disposition, les chefs d'entreprises sont en général propriétaires des lieux où ils travaillent. C'est là un progrès notable dans la mesure où, antérieurement, $5 \%$ seulement des artisans de la médina étaient propriétaires de leurs ateliers (selon un recensement réalisé en 2005 par la Délégation régionale de l'artisanat). Pour les 95\% restant, la location était la règle. Les unités installées à Aïn Nokbi sont modernes, et leurs locaux spacieux; elles sont donc en mesure d'améliorer (quantitativement et qualitativement) la production et, en s'appuyant sur une demande de plus en plus différenciée, d'entretenir un processus de croissance de l'activité ainsi que de création d'emplois sur le territoire.

Cette dynamique favorable, on l'a vu, est le fait principalement de maîtres artisans, ex-patrons d'ateliers dans la vieille ville. Mais d'anciens ouvriers artisans (sana'a, en arabe) y contribuent significativement, notamment ceux qui exerçaient dans les ateliers de la Place Lalla Yedouna. Ayant bénéficié de subventions, ces derniers, organisés en collectifs de deux ou trois personnes, se sont installés à leur propre compte, certains comme locataires, d'autres comme propriétaires des locaux. Forts de leurs savoir-faire (acquis dans la vieille ville), ces groupes d'artisans entrepreneurs sont entrés dans des réseaux de production au sein desquels ils mutualisent leurs ressources tout en limitant les risques qu'ils prennent. D’autres anciens salariés des ateliers de la médina (dont le nombre est difficile à établir) se sont également installés à leur compte, soit à Aïn Nokbi, soit dans d'autres zones à la périphérie de Fès.

$\mathrm{Au}$ total, on peut donc en conclure que, sous l'effet de la politique publique de redéploiement des activités hors médina, toute une dynamique d'entreprises est à l'œuvre sur le territoire de Fès, portée non seulement par des ex-patrons d'ateliers de la vieille ville, mais aussi par leurs anciens salariés (ayant bénéficié d'un apprentissage par la pratique), ce qui indique qu'une relève entrepreneuriale est en cours et qu'elle devrait permettre, à terme, de tirer profit pleinement du potentiel de savoirfaire disponible localement. Efficacement mobilisés par les entreprises installées dans la nouvelle zone d'activités, ces savoir-faire ouvrent de belles perspectives, surtout qu'il existe, à Fès, une main-d'œuvre nombreuse qui possède, en outre, des qualifications polyvalentes appréciables acquises dans le cadre du système traditionnel de transmission familiale des métiers.

Ceci dit, notre enquête fait apparaître un énorme retard dans la mise en route effective des activités à Aïn Nokbi. Ainsi, 111 projets (soit 25\% du total) n'ont à ce jour pas démarré, faute de locaux n'ayant pas encore été construits en raison de la faiblesse des moyens financiers dont disposent les porteurs de ces projets, lesquels sont d'autant plus contraints qu'ils assument en général seuls-ou avec leur famille-les risques qu'ils prennent en s'engageant dans un projet de création ou d'extension d'activité.

La proximité est la caractéristique la plus appréciée en médina. Elle s’entend d'abord au sens habituel d'un accès rapide aux matières premières et de contacts facilités entre artisans, comme entre ces derniers et leurs clients, fournisseurs et sous-traitants, avec les effets positifs qui en découlent en termes de coûts de production et de transaction. Mais la proximité en médina s'entend aussi au sens de petit trajet (voire pas de trajet du tout pour ceux qui exercent chez eux ou juste en dessous de chez eux) entre le lieu de 
travail et le lieu d'habitation, ainsi que de disponibilité tout près de soi, en permanence, de produits de consommation courante et de services divers (réparation, restauration, etc.). En revanche, à Aïn Nokbi, l'avantage de la proximité n’est pas (encore) avéré : ainsi beaucoup d'artisans qui y sont installés se plaignent d'être loin de leurs fournisseurs, sous-traitants et clients, loin également de leurs lieux de résidence alors même que les services nécessaires au quotidien (commerces, restauration...) n’y sont pas disponibles en quantité et qualité suffisantes. Aussi, la localisation à Aïn Nokbi est-elle perçue négativement, d'autant que l'éloignement géographique se conjugue avec le manque d'équipements publics, comme les transports collectifs ou les services de sécurité.

Si ces difficultés sont réelles, elles ne bloquent cependant pas la dynamique. Il est permis de penser que, grâce aux nouvelles zones artisanales auxquelles elle a donné naissance, la politique de redéploiement spatial des activités à Fès est susceptible de contribuer à une pérennisation des métiers et, au-delà, à une relance du dynamisme industriel local à partir de l'artisanat.

\section{Une dynamique d'innovation de produits et de procédés, source d'amélioration de la qualité}

Phénomène relativement ancien, la raréfaction du foncier destiné à la production au sein de la médina était arrivée, au début des années 2000, à un point tel que les nouveaux artisans ne pouvaient plus s'installer dans la zone traditionnellement réservée à leur corporation. Ainsi en a-t-il été de nombreux dinandiers établis au cours des dernières décennies qui, faute de place dans le quartier des Essafarine (dinandiers, en arabe), ont dû se redéployer vers d'autres parties de la vieille ville. En d'autres termes, ces dinandiers arrivés tardivement se sont retrouvés dispersés un peu partout dans l'espace de la médina, ce qui, d'une part, les privait du bénéfice des économies d'agglomération et, d'autre part, plus globalement, les empêchait d'avoir une bonne vision de l'évolution de leur profession. Aujourd'hui, sur le nouveau site d'Aïn Nokbi, le regroupement spatial des unités de production (selon une logique de proximité sectorielle) est de nouveau à l'œuvre, ouvrant la voie à terme à des économies externes d'agglomération, ainsi qu’à des économies de spécialisation (grâce à une division de travail entre les unités), synonymes de productivité élevée.

Autre point à souligner, la concurrence sur le site de la nouvelle zone d'activités d'Aïn Nokbi est fondée davantage sur la qualité des produits (et donc sur l'innovation) que sur des salaires bas et des conditions de travail précaires. Cette évolution positive n'est pas sans relation avec le nouveau profil des entreprises regroupées sur ce site, dans la mesure où la politique de transfert a induit une refonte de la structure organisationnelle des unités économiques déplacées, une amélioration sensible de leurs modes de gestion, une extension de leur recours aux technologies modernes, etc. Ces différents changements, en favorisant une élévation de la qualité des produits, une meilleure compétitivité et une plus grande réactivité face au marché, ont contribué à donner une nouvelle impulsion à la filière de la dinanderie. Impulsion d'autant plus remarquable d'ailleurs que, parallèlement, la production dinandière s'est diversifiée, au sens où des objets modernes (ou plus exactement à usage répandu aujourd'hui comme les poignets ou les vasques) ont été ajoutés à la panoplie d'articles fabriqués traditionnellement (plateaux de diverses formes et dimensions, bougeoirs, théières, etc.). Au total, c'est donc à un approfondissement du processus d'innovation que nous assistons, quant aux produits et aux procédés fabrication, mais aussi à l'organisation et à la gestion. 


\section{Les retombées sur le triple plan social, environnemental et culturel}

L'évaluation de la politique marocaine de délocalisation des activités artisanales des vieux centres urbains vers de nouvelles zones d'activités ne doit pas reposer seulement sur des critères d'ordre économique. Elle doit porter aussi sur des critères d'ordres social (revenus, niveau de vie, santé...), environnemental (diminution des rejets polluants) et cultuel (transmission des savoir-faire dans le temps, maintien des traditions de coopération et de cohésion sociale...), ces derniers aspects étant aussi cruciaux (voire davantage) que les volumes de production et les résultats financiers.

\section{L'impact de la délocalisation en termes de revenus des artisans et de débouchés pour leur production}

S’agissant de l'impact sur les revenus, il faut clairement distinguer deux groupes d'artisans: ceux qui sont restés dans la vieille ville et ceux ayant opté pour la délocalisation. Parmi les premiers, 78\% déclarent avoir enregistré une baisse d'activité estimée à $60 \%$ ou plus, avec les incidences qu'on imagine sur leurs revenus. Les causes de cette baisse sont multiples. D’abord, personnellement concernés par le transfert ou pas, tous les artisans ont dû cesser leur activité pendant le déménagement. Ensuite, les nombreux retards dans l'installation à Aïn Nokbi ont provoqué des ruptures de la chaîne de production qui, compte tenu des interdépendances entre les deux groupes d'artisans, ont beaucoup entravé la remise en route des ateliers restés dans la médina. Enfin, le départ vers le nouveau site d'une partie importante des artisans dinandiers de la vieille ville ne met pas fin aux nombreuses relations (notamment de sous-traitance) qu'ils entretiennent avec leurs collègues restés dans l'ancien. Le véhicule utilitaire étant le moyen de transport privilégié entre les deux sites, les coûts de ce service ont beaucoup augmenté, ce qui a induit une hausse du prix de revient pour tous. À titre indicatif, le transport de $100 \mathrm{~kg}$ de matière de la médina vers Aïn Nokbi coûte $50 \mathrm{dh}$, alors qu'au sein de la vieille ville, le même service (effectué souvent à dos d'âne) ne coûte que de 5 à 10 dh.

En ce qui concerne le deuxième groupe de dinandiers (ceux installés à Aïn Nokbi), leur situation est beaucoup plus encourageante. D'une part, ils apprécient positivement leurs nouvelles conditions de travail, ainsi que les occasions que leur offre leur nouvel espace pour moderniser leur processus de production et l'organisation de leurs ateliers. D'autre part, l'installation à Aïn Nokbi, en leur assurant une meilleure lisibilité et une reconnaissance officielle, leur ouvre des possibilités de mobiliser les moyens (financiers, techniques...) dont ils ont besoin pour améliorer à la fois leurs équipements, leurs qualifications, la qualité de leurs produits et leurs niveaux d'activités et de revenus. Sans doute, les problèmes liés à l'installation n'épargnentils pas ce deuxième groupe d'artisans. Sur leur nouveau site, plusieurs conditions nécessaires au bon lancement de leurs affaires ne sont pas réunies. En particulier, les services collectifs de base (transport, santé...) y font cruellement défaut, comme cela a été signalé ci-dessus. Plus handicapant encore, la sécurité des biens comme des personnes n’y est pas assurée de jour comme de nuit, faute de présence permanente des institutions publiques compétentes. Les chefs d'ateliers que nous avons interviewés relatent unanimement les difficultés qu'ils rencontrent pour faire face à l'insécurité, qui se manifeste notamment par des actes d'agression physique pour vol et pour viol (s’agissant du personnel féminin). 
Deux sortes de mesures sont préconisées par les chefs d'entreprises pour rendre véritablement bénéfique leur installation à Aïn Nokbi: d'une part, inciter le plus possible de dinandiers encore réticents à accepter le transfert, de façon à favoriser sur le nouveau site un maximum d'effets d'agglomération; et, d'autre part, suppléer aussi rapidement que possible au manque de services dans la nouvelle zone d'activités: commerces, espaces de restauration et, surtout, poste de police pour assurer un minimum de sécurité. Ces deux catégories de mesures font écho aux deux inconvénients massivement dénoncés, à savoir l'éloignement par rapport aux partenaires professionnels, d'une part, et l'insécurité, d'autre part. En outre, se pose de façon cruciale le problème des déplacements des artisans entre leur foyer et leur lieu de travail à Aïn Nokbi. Il convient de le traiter urgemment, au moyen d'une desserte régulière de la zone dans le cadre du réseau de transport interurbain.

Dernier problème, mais non le moindre: dans sa configuration actuelle, le site d'Aïn Nokbi présente de réels avantages comme zone de production, mais il est très peu adapté pour attirer et accueillir la clientèle. La majorité des artisans interrogés, bien qu'assumant le choix qu'ils ont fait de déménager leur atelier, déplorent fortement d'avoir perdu leurs débouchés traditionnels en s'éloignant de la médina, grand centre de commerce et d'attraction touristique. Pour y remédier, certains artisans vont jusqu'à envisager de racheter un petit local dans la vieille ville pour retrouver leur zone de chalandise. C'est en tout cas ce qui ressort des propos recueillis lors de notre enquête, plusieurs répondants ayant souligné la nécessité d'éviter le risque d'une rupture définitive du rapport direct avec leurs clients, au bénéfice des commerçants qui, en général, sont intéressés uniquement par le profit.

À cet égard, la suppression des intermédiaires est nécessaire à la fois pour permettre un écoulement plus fluide des produits artisanaux et de meilleurs revenus aux artisans. De même, le directeur de la Délégation régionale de l'artisanat (à la suite d'une réunion que nous avons organisée avec lui) a proposé la création à Aïn Nokbi d'un atelier témoin (appelé DAR ENHASS) voué à l'exposition permanente des divers articles de dinanderie affichant clairement les noms de leurs producteurs. Cet atelier témoin, qui sera placé sous l'égide des artisans concernés, aura vocation de favoriser la promotion des produits en facilitant leur écoulement, aussi bien à l'interne qu'à l'externe.

Ceci dit, il faut se garder de toute dramatisation excessive de la situation. Globalement, la politique de délocalisation vers la zone d'Aïn Nokbi continue d'avoir la faveur des artisans concernés. À terme, estiment ceux-ci, la délocalisation ne manquera pas d'avoir des effets bénéfiques sur leurs niveaux d'activités et de revenus grâce, d'une part, aux possibilités d'extension de leur capacité productive qu'offre le nouveau site et, d'autre part, à la conversion, au sein de la médina, des espaces désormais libres en lieux de vente, permettant aux artisans d'exposer leurs produits dans de meilleures conditions.

\section{L'impact de la délocalisation sur les conditions de travail et l'environnement}

Concernant les conditions de travail, la comparaison des deux sites ne laisse place à aucun doute, tant le progrès que représente le nouveau site par rapport l'ancien est énorme. Dans la médina, on le sait, l'exiguïté de l'espace est aggravée par la dégradation 
du bâti, l'absence d'éclairage et d'aération dans les ateliers, le tout se traduisant par une grande pénibilité et une forte exposition de la main-d'œuvre aux accidents de travail, non seulement lors de la manipulation des machines, mais aussi lors des déplacements.

Par contraste, les avantages d'Aïn Nokbi sont nets sur ce plan. Situés dans des bâtiments neufs et solides, les ateliers sont spacieux, bien aérés, correctement éclairés, etc. Munis d'équipements modernes (eau courante, toilettes, parfois douches), ils offrent un cadre de travail nettement amélioré en termes d'hygiène et de sécurité et, plus largement, des conditions de travail beaucoup moins pénibles. Ce que confirme le témoignage suivant recueilli auprès de l'employé d'un artisan dinandier installé dans la nouvelle zone:

«On exerçait le métier dans des conditions pires que celles des animaux, on était noir à cause des déchets; personnellement, je travaillais dans un réduit de $2 \mathrm{~m}^{2}$, je n'avais pas suffisamment de hauteur pour me mettre debout... c'étaient des conditions de travail indignes des êtres humains... Aujourd'hui, à Aïn Nokbi, c'est autre chose, nous travaillons dans un environnement propre, les bâtiments sont beaux... vraiment rien de comparable entre la nouvelle et l'ancienne situation, on a retrouvé la dignité d'êtres humains.»

Même constat quant à l'environnement, relativement mieux préservé sur le nouveau site grâce à une diminution du taux global de pollution de l'Oued El Jaouahir et à la mise en place de procédés industriels de traitement des eaux au sein même des unités de production, les entreprises ayant réalisé les travaux nécessaires selon les normes internationales. Toutefois, il importe de le noter, la préoccupation écologique et paysagère est totalement absente dans la conception initiale du site par la société Al Omrane, au point qu'il n'existe actuellement à Aïn Nokbi ni plantation, ni espace vert d'aucune sorte; de même qu'on n'y trouve aucun espace de convivialité (lieu public de rencontre, de détente, de consommation). Il en résulte un site industriel sans âme, froid et peu attrayant, pour la population autochtone comme pour les touristes.

\section{L'impact sur le système local de valeurs: modernisation des méthodes de travail, mais maintien (au moins en partie) des traditions de coopération}

Depuis plusieurs décennies, l'activité de la dinanderie connaît une évolution importante qui affecte les rapports sociaux en son sein, ainsi que les conditions de reproduction de la corporation et la place particulière qu'y tient la famille. En fait, on observe une régression du rôle de la famille dans le processus de production, notamment dans les unités les plus affectées par la baisse de la demande, par l'exiguïté des lieux de travail et, enfin, par la pollution croissante en médina. Dans ces ateliers en difficulté, faute de perspectives favorables, les chefs d'entreprises n'impliquent plus, autant que par le passé, les membres de leurs familles. À l'inverse, dans la nouvelle zone d'activités, l'emploi familial a fait une réapparition (en particulier dans les entreprises d'une certaine taille) non seulement dans les tâches de production, mais aussi d'administration. Ainsi, lors de l'enquête, nous avons eu affaire à quatre entreprises dans lesquelles ce sont les propres filles des patrons, toutes diplômées de l'université, qui sont chargées de la 
comptabilité et plus généralement de la gestion de l'entreprise, ce qui tranche avec les pratiques au sein de la médina, où les enfants sont sollicités surtout pour les opérations de production, la gestion restant le domaine du chef de famille.

Ceci étant, qu'ils aient opté pour le nouveau site ou préféré rester dans l'ancien, les dinandiers de Fès ont une perception positive de leur métier, au point de vouloir majoritairement le transmettre à leurs enfants. Toutefois, l'apprentissage, qui reste une pratique courante dans la médina, est étonnement négligé à Aïn Nokbi. Ainsi, au sein des 80 entreprises étudiées, nous n'avons recensé que 45 apprentis. Interpellés sur ce point, les chefs d'entreprises invoquent deux raisons en guise d'explication: d'une part, l'application stricte, dans la nouvelle zone, de la législation qui interdit le travail des enfants de moins de 15 ans; et, d'autre part, les perspectives défavorables de certains métiers dont la dinanderie, qui provoquent une perte de motivation chez les jeunes. Ce recul de l'apprentissage a été, d'une certaine manière, compensé par un recours accru au travail féminin, dans les ateliers. Aussi les femmes constituent-elles, aujourd'hui, un acteur à part entière jouant un rôle non négligeable dans l'activité dinandière à Aïn Nokbi (49\% des ateliers du site comprennent des personnes de sexe féminin parmi leurs salariés). Dans leur majorité, ces femmes effectuent des tâches secondaires : différents travaux de nettoyage nécessaires à plusieurs étapes du processus de production, aux moments du polissage et du traitement de surface et de la finition. Cela explique qu'elles soient maintenues dans un statut inférieur qui les cantonne à des tâches souvent pénibles et peu valorisantes.

Dernier point à souligner quant à l'impact sur le système des valeurs: dans la dinanderie (comme dans d'autres artisanats ancestraux), on constate un certain affaiblissement des structures communautaires. En raison notamment de la dispersion spatiale des membres de la corporation, les possibilités d'entraide sont amoindries, d'autant plus que la logique ancienne des liens de parenté a plus de mal à s'imposer et que les chefs de famille ont plus de difficulté à exercer leur autorité. À la question de savoir si la délocalisation des activités a affaibli le système local de valeurs traditionnelles, les artisans interrogés répondent "oui» à 93\%: les effets majoritairement déplorés sont la dégradation du lien social et la perte de la convivialité du voisinage. En revanche, l'appartenance à la communauté fassie continue d'être un facteur de solidarité entre acteurs économiques locaux. Grâce au système de valeurs qui fonde cette communauté (respect de l'autre, confiance mutuelle, réciprocité), ceux qui en font partie soit la majorité des dinandiers de Fès continuent de cultiver entre eux divers modes de coopération: échanges d’information, prêts mutuels de matériel et de matières premières, efforts partagés pour améliorer la qualité des produits. Si bien qu'au total, à Aïn Nokbi comme dans la médina, les entreprises de dinanderie, tout en étant naturellement en concurrence les unes avec les autres, maintiennent entre elles des relations d'entraide qui ne contribuent pas peu à la vitalité de l'activité dans son ensemble.

\section{Conclusion}

Dans la médina de Fès, la dynamique économique à base artisanale s’appuie largement sur les savoir-faire ancestraux, la polyvalence de la main-d'œuvre et des équipements, ainsi que les liens de coopération entre acteurs (favorisés par la proximité spatiale). Mais elle repose davantage encore sur une pression forte et constante, exercée sur la main-d'œuvre: faibles rémunérations, conditions de travail précaires, etc. Par ses 
incidences en termes de démotivation des ouvriers et des apprentis, cette pression finit cependant, contradictoirement, par influencer négativement la productivité et la qualité des produits. Dans la nouvelle zone d'activités d'Aïn Nokbi, les choses semblent aller différemment: parce que la main-d'œuvre y est considérée comme le facteur-clé de la dynamique, la performance économique se fonde sur des salaires décents et des conditions de travail (relativement) satisfaisantes. L'effet attendu en est une plus grande motivation des employés, incités de la sorte à déployer leur créativité et leurs capacités d'innovation (sur les plans technologique et organisationnel) non seulement pour produire davantage, mais aussi pour améliorer sans cesse les méthodes de fabrication et la qualité des produits.

Si le processus engagé est mené à son terme, il ne manquera pas d'avoir des effets bénéfiques non seulement sur la dinanderie, mais aussi sur d'autres métiers artisanaux dans l'ensemble du territoire de Fès. Mais encore faut-il que les équipements nécessaires soient construits à Aïn Nokbi et dans les autres zones d'activités et que, graduellement, le transfert touche de plus en plus de segments des chaînes de production. À défaut de quoi, les nouvelles zones seraient peu attrayantes aux yeux des maîtres artisans de la médina, pour lesquels la délocalisation ne présenterait alors aucun intérêt. À cet égard, la mise en place, dans les nouvelles zones, de services de proximité (santé, sécurité, transport, etc.) doit être conçue de façon à favoriser un effet d'entraînement en chaîne. S'agissant spécifiquement de l'artisanat ancien, il importe de l'envisager en conciliant tradition et modernité. Les pouvoirs publics doivent donc agir notamment pour préserver les droits de propriété des artisans (qui, contrairement à l'idée reçue, innovent constamment) en assurant la protection des innovations par des brevets, et des créations artistiques par des droits de propriété intellectuelle. Cette action doit se combiner avec une promotion intelligente des productions artisanales locales ciblant la clientèle touristique, en particulier étrangère, afin de redonner à l'artisanat de Fès son rayonnement international d'autrefois. 
Tableau 1 Récapitulatif des unités productives enquêtées dans la dinanderie du territoire de Fès

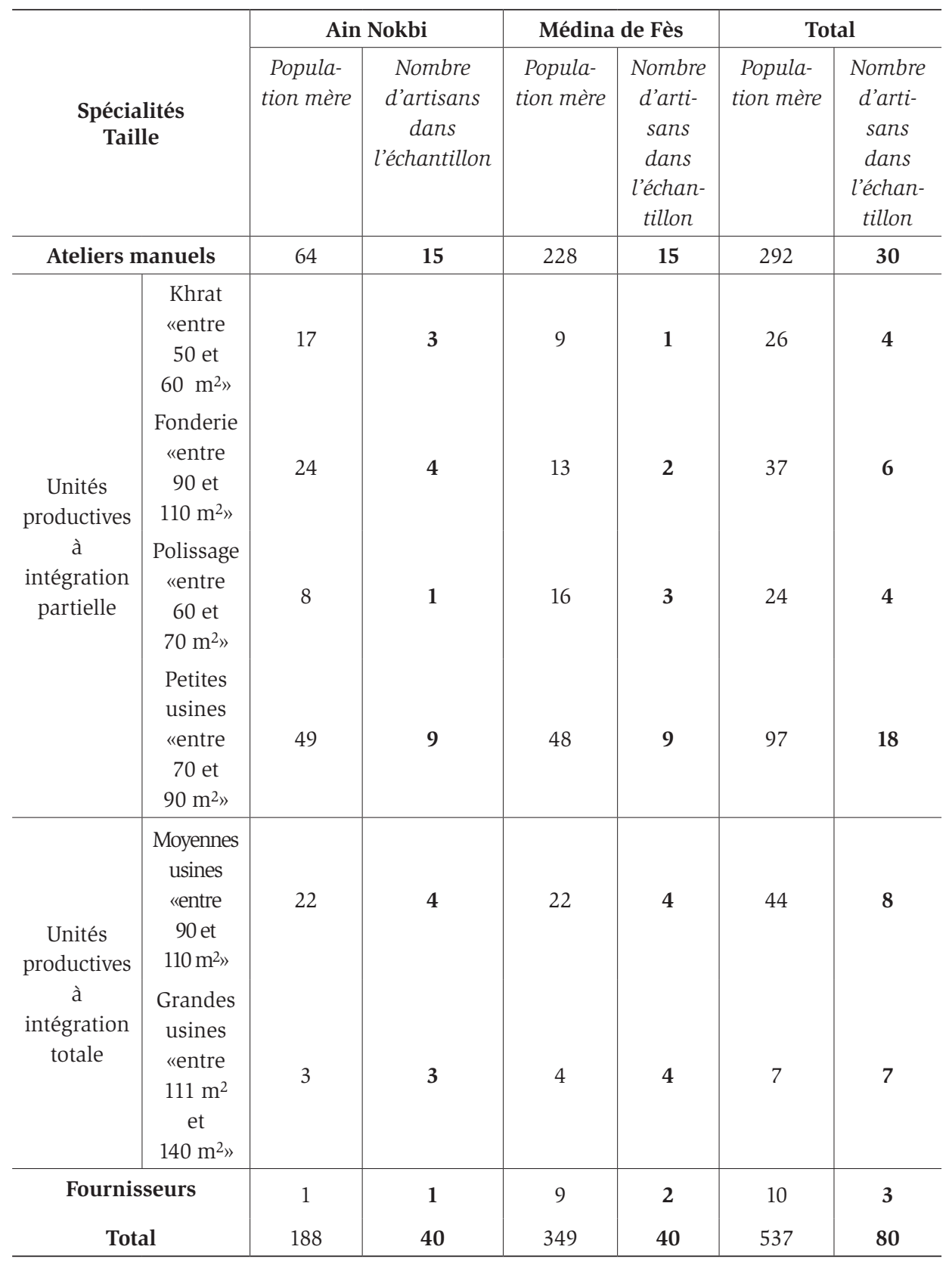




\section{Bibliographie}

BECATTINI, Giacomo (1992) Le district industriel: une notion socio-économique, dans Georges Benko, Alain Lipietz (dir..), Les régions qui gagnent. Districts et réseaux: les nouveaux paradigmes de la géographie économique, Paris, PUF, p. 35-55.

BUOB, Baptiste (2009) La dinanderie de Fès: un artisanat traditionnel dans les temps-moderne-Une anthropologie des techniques par le film et le texte, Maison des sciences de l'homme.

CHAMBRE DE L'ARTISANAT DE FÈS (2005) Recensement des entreprises artisanales de dinanderie à Fès, Fès.

COURLET, Claude et PECQUEUR, Bernard (1996) Districts industriels, systèmes productifs localisés et développement, dans Lahsen Abdelmalki, Claude Courlet (dir.), les nouvelles logiques de développement, L'Harmattan, Paris, p. 91-102.

FEJJAL, Ali et GUERRAOUI, Driss (1988) L'industrialisation de l'artisanat à Fès, dans Histoires de développement, Cahiers de l'IES, Lyon, décembre, nº 4, p. 6-8.

FERGUENE, Améziane (2007) Entreprises artisanales et dynamiques locales dans les médinas de Fès (Maroc) et de Sfax (Tunisie). Critique économique, Rabat, hiver-printemps 2007, no 19, p. 109-129.

FERGUENE, Améziane (2001) Savoir-faire artisanaux et dynamismes locaux dans les vielles villes du Maghreb: l'exemple de la Médina de Sfax. Dans A. Sassu (dir.) Savoir-faire et productions locales dans les pays de la Méditerranée. Paris, Publisud, p. 271-293.

GREFFE, Xavier et SIMONNET, Véronique (2008) La survie des entreprises culturelles: le rôle du regroupement géographique, dans Recherches Économiques de Louvain, vol.74, nº3, p. 327-357.
LE TOURNEAU, Roger (2009) Fès avant le Protectorat. Étude économique et sociale d'une ville de l'occident musulman, (dernière édition), La Porte, Cop., Rabat, 1987, 670 p. (la version initiale est parue en 1949).

MASSIGNON, Louis (1925) Enquête sur les corporations musulmanes d'artisans et de commerçants au Maroc (1903-1924), Paris, Ernest Leroux.

SCHMITZ, Hubert (1990) Petites entreprises et spécialisation souple dans les pays en développement, dans Travail et Société, vol.15, no3, p. 271-305. 